Zhu, D., Zhang, X., Fu, L., Wang, P., Zhai, S. (2018). A Pilot Study on Real-time Monitoring of Heart Rate and Movement Speed in Middle-distance Race of Physical Education Classes. Journal of Educational

Technology Development and Exchange, 11(1), 35-44

\title{
A Pilot Study on Real-time Monitoring of Heart Rate and Movement Speed in Middle-distance Race of Physical Education Classes
}

\author{
Daqing Zhu \\ Tsinghua University, Hubei University of Arts and Sciences \\ Xingui Zhang \\ Tsinghua University \\ Lanying Fu \\ Zhengzhou Shengda Economic and Trade Management College \\ Peiyong Wang \\ Tsinghua University \\ Shaohong Zhai \\ Xi'an Physical Education University
}

\begin{abstract}
In Chinese universities, students need to participate in the middle-distance-race. Normally, female students are required to participate in the race of 800 meters, while male students are required to participate in the race of 1000 meters. However, it is difficult for teachers to grasp the real time information of students during the race. And there is a lack of timely communications between the teachers and students. Focusing on this issue, this study, with the use of POLAR heart rate sensor and other modern information technologies, expands the original function of the sensor to achieve a concurrent operation of detecting heart rates and automatically measuring the movement speed. The researchers have successfully designed a micro system to monitor the process of middle-distance race. Moreover, the study also engages in a preliminary experiment verification so as to provide object and effective reference and basis for the middle-distance race physical education teaching in universities.
\end{abstract}

Keywords: Real-time Monitoring, Physical Education Teaching, Middle-distance Race, Exercise Heart Rate, Sensor, Movement Speed

\section{Introduction}

To amend the situation in which a continuous decline occurring to the physical health of students, the Central Committee of the Communist Youth League of China and the State Sports General Administration of China together launched a program entitled "Sunshine Sports for Hundreds of Millions 
of Students" in 2007. In this context, the Sunshine Sports Middle-distance Running Program has started. This program is of profound significance to fully carry out the education policy made by the Communist Party of China, to promote an active engagement of college students in the exercise, and to improve their overall endurance quality and physical fitness and health. This program has embraced a gratifying achievement. But in the meanwhile, some issues have exposed. For example, injuries, even sudden deaths have frequently occurred in the middle-distance running physical education classes in the universities. In such classes, female students normally participate in the race of 800 meters, and male students usually join in the race of 1000 meters. The issues have aroused a deep concern. As a result, the public has started to pay attention to the monitoring of the running process, focusing on the scientific nature of exercise.

Exercise load monitoring methods used in the professional trainings have gradually been applied in public sports and become popularized. The monitoring approaches are constantly enriched. And monitoring devices, such as wristbands and wrist monitors are steadily coming into the world (Yang et al., 2014; Ji et al., 2015; Jiang et al., 2015; Neurosky, 2018). However, these monitoring devices are applicable only to the self-monitoring of single individuals, and lack sufficient monitoring indicators. The circumstances in universities are far more complicated, as there are a large number of students participating in the middle-distance running physical education classes at the same time. Thus, these devices are incompetent to satisfy the demand of real-time monitoring on the mass students. In other words, the sports loading supervision system of the middledistance running physical classes has not yet been systematically established.
Starting from the perspective of cluster monitoring, this study employs the shortrange wireless transmission technology, which enables us to monitor the heart rates during exercise, as well as the speed and distance of motion. In addition, the relevant analysis of these indicators can be performed. Consequently, this study achieves the realtime detection of heart rate and motion speed, as well as the data transmission. Thereby, it resolves the problems related to real-time cluster monitoring for the vast number of runners. It can also provide an early warning for those who may get injured.

\section{Composition of Exercise Heart Rate and Motion Speed Monitoring Equipment}

With a purpose to monitor the longdistance running in the athletic field at universities, a local area network has been equipped to the system. A synchronous monitoring on both the heart rate and motion speed during the long-distance running has also been designed and achieved base on the use of Polar heart rate sensor. Then, the realtime remote monitoring is realized through the wireless relay. This set of micro system is of strong practical value for the safety monitoring of the long-distance running process, the reasonable adjustment of the amount of exercise, and the control of exercise intensity. This system emphasizes on the scientific nature of exercise.

\subsection{Characteristics of the Polar Heart Rate Sensor}

There are quite a number of companies engaged into the research on the heart rate acquisition in the process of movement, of which the Polar is the most renowned one over the world. This company has produced mature products (Lows \& Ólaighin, 2014; Yokota, 2015; Augustyniak, 2011; Han et al., 
2017). Polar WearLink heart rate sensor is one of them (as shown in Figure 1).

This model of heart rate sensor is equipped with the following main features: 1) The sensor is equipped with a comfortable, light, and flexible elastic bandage; 2) The signal transmitted by wireless employs code encryption technology, which greatly improves the reliability of the signal during wireless transmission; 3) The sensor uses a 3-volt lithium of CR2032 model battery, with an average service life up to 300 hours. This battery is replaceable. It is generally in a sleep state and activated only during operation, thus the amount of power consumption is tiny; 4) The whole product is waterproof, hence it is allowed to work 30 meters beneath the water; $5)$ The elastic bandage can be removed off to clean; 6) The lock connected at the front-end makes it easier for users to install and remove sensors; 7) The sensor has a price advantage.

Considering the superiorities of the Polar Wear Link sensor described above, the researchers of this study adopt it as the heart rate sensor in the heart rate collection and wireless emission system. In this way, the time for development has been saved in an effective manner. Moreover, this mature sensor product also promises a good consistence in utility so as to ensure a smooth implementation for the subsequent development.

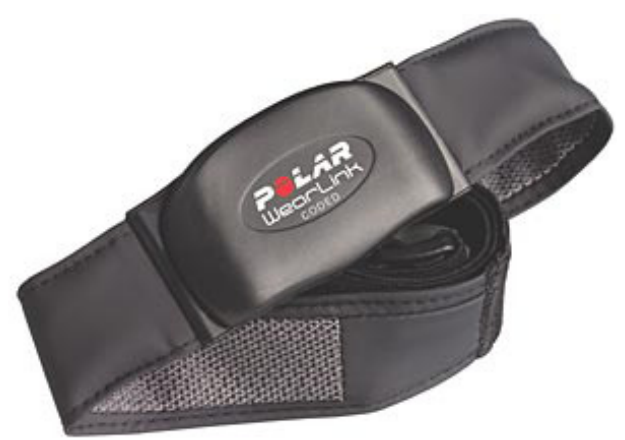

Figure 1. The Polar heart rate sensor

\subsection{Standard Athletic Field and the Location for Receiver Placement}

The standard internal track of the athletic field, namely, the first lane, should be built to 400 meters. It consists of two straight roads and two bent roads, and also can be understood as consisting of four equal parts of 100 meters (as shown in Figure 2).

The heart rate sensor needs to be put on the runners. And four heart rate wireless relays are placed at the junctions between the straight roads and the bent roads. And these wireless relays complete the information transmission in an automatic manner when the runners pass through each relay. The wireless relays forward the signals of the heart rate received from the heart rate sensor and the time information in real time to the computer at remote for processing. And then the realtime monitoring on the runners is actualized based on three major parameters, namely, the heart rate, distance, and time. In addition, several indicators, such as the current exercise amount, exercise load, exercise stimulating intensity, energy consumption, cardiac blood output, and heart rate variability can be computed out. 


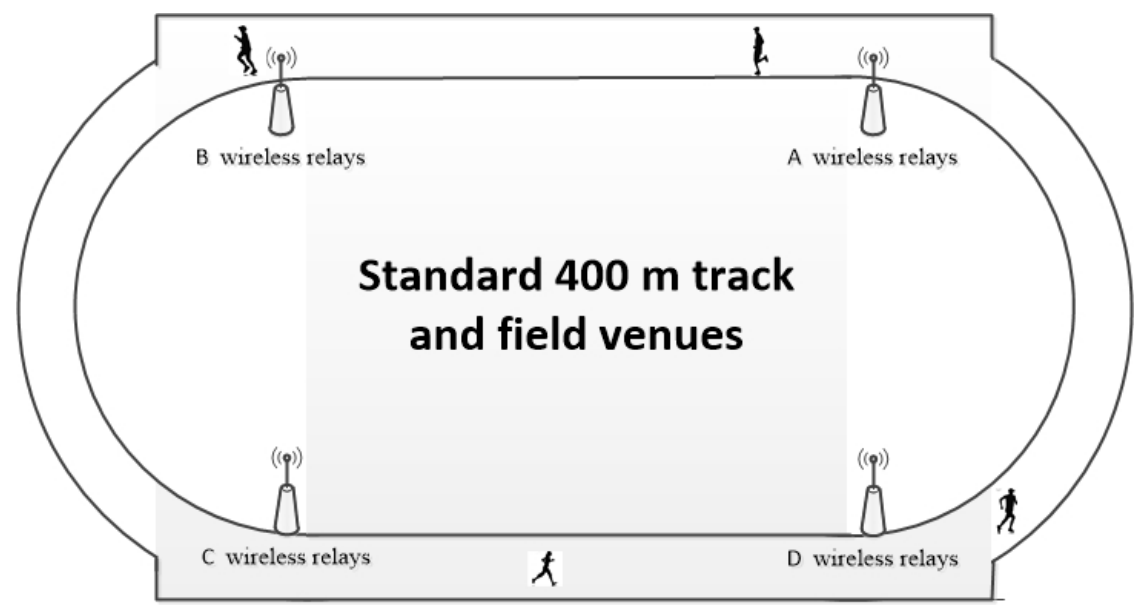

Figure 2. The location of the heart rate wireless receiver in a standard athletic field

\section{Design and the Working Principles of the Monitoring Related Equipment in Middle- Distance Running Physical Education Class}

\subsection{Features of the Wireless Signal Transmission of Polar Heart Rate Sensor}

The function of the Polar heart rate sensor is to detect the electro-cardiac signal and measure the interval between the $\mathrm{R}$ waves (the time between two adjacent electrocardiac R waves) through the ECG signal, so as to calculate the value of heart rate corresponding to each minute. This heart rate value per minute reflects the immediate heart rate. The average heart rate can be obtained after processing immediate heart rate data by software filtering method.

The data emission of the Polar heart rate sensor is performed in a wireless way. With the NRF2401 wireless chip serving as the core, the data emission is conducted at the device of radio frequency transceiver for heart rate acquisition and processing which is located in the $2.4 \mathrm{GHz}$ ISM band, with a wireless frequency ranging between 2400 to $2524 \mathrm{MHz}$.
In the ShockBurstTM transceiver mode, the data frame delivered via wireless processes the header and CRC checking code in an automatic pattern. At the time that the data is under receiving, the header and CRC check code are automatically removed. While at the time that the data is in sending, the header and CRC check code are automatically added.

Regarding to the wireless data frames, there are 26 valid bytes contained in each frame of the data. And two key data, including 8 bit immediate heart rate and 8 bit average heart rate are embodied in these 26 valid bytes.

The maximum communication rate of $1 \mathrm{Mbps}$ and 125 channels armed to the chip allows it to satisfy the needs of frequency hopping communication and multipoint communication. The chip is configured with words of 120 bits ( 15 bytes). The configuration words are realized through software settings and are featured with great flexibility. In this example, the emission power and the operating frequency contributes to the two important parts, shown as in Tables 1 and 2. 
A Pilot Study on Real-time Monitoring of Heart Rate and Movement Speed in Middle-distance Race of Physical Education Classes

Table 1. Setting for four-speed emission power

\begin{tabular}{|c|c|c|}
\hline \multicolumn{3}{|c|}{ RF OutPut Power } \\
\hline Bit 9 & Bit 8 & Power \\
\hline 0 & 0 & $-20 \mathrm{dBm}$ \\
\hline 0 & 1 & $-10 \mathrm{dBm}$ \\
\hline 1 & 0 & $-5 \mathrm{dBm}$ \\
\hline 1 & 1 & $0 \mathrm{dBm}$ \\
\hline
\end{tabular}

Table 2. Settings for operating frequency and working mode

\begin{tabular}{|l|l|l|l|l|l|l|c|}
\hline \multicolumn{5}{|c|}{ RF CH\# Select bit } & RXEN(bit0) \\
\hline bit7 & bit6 & Bit5 & Bit4 & Bit3 & Bit2 & Bit1 & 1 or 0 \\
\hline
\end{tabular}

In the 120-bit configuration words, the bit7-bit1 works as the selection bit for channels. It can be chosen within the range of 125 channels, with a channel spacing of $1 \mathrm{MHz}$. The calculation method of the wireless working frequency is as follows: channel frequency $=2400 \mathrm{MHz}+\mathrm{RF} C \mathrm{CH} \# \times 1 \mathrm{MHz}$. The Bit0 is the RXEN selection bit: set to 1 for the wireless data reception mode and set 0 to the wireless data transmission mode.

The power configuration for the Polar heart rate sensor brings us the possibility to realize our assumption of monitoring the heart rate and measuring the distance at the same time. The best setting seems to be that the reception device captures the data frames of the heart rate sensor when the runner is within the range of 4 meters from each 100-meter junction. When runner runs through the connection points each time, the receiver (wireless relay) placed at the junction can be refreshed so as to represent that he or she once passes this place at a certain moment. The receiving device loses its connection with the heart rate sensor when the runner is out of the range of 4 meters away from the junction point between the 100 -meter roads.

\subsection{The Principle of Polar Heart Rate Sensor Concurrently Achieving Distance Measurement}

As mentioned earlier, four wireless heart rate relays are placed at the junctions of the straight road and the bent road (also at the junction of four 100 meters). At the time that the runner passes through each wireless relay, the relay receives data frame of the heart rates sent from the heart rate sensor, as well as the information about the time the runner reaches the relay. From the perspective of power saving, we suggest setting the wireless transmission power of the Polar heart rate sensor at the lowest level. Under this circumstance, the actual reliable transmission distance is 3 to 4 meters. With such setting, the time error for obtaining data through the relay is small enough to meet the monitoring requirements of long-distance running.

\subsection{Wireless Relays and Real-Time Follow- up Treatment of Heart Rate Sensor}

It should be noted that the power design of the relays requires a full-aspect consideration (Zhang et al., 2015). The relays are to be placed in the athletic field, and generally supplied by batteries. Therefore, the 
over-strong power for wireless emission is a waste. But the over-small power can cause the computer at the terminal fail to receive data. For this end, when setting the emission power of the relays, we need to consider in a comprehensive way, taking into account the power supply, the height of the relay placement, the performance of the antenna, as well as the factor of greater importance, that is, the distance of the subsequent remote processing equipment, environmental factors, etc.

Nowadays, there are a variety of types of wireless modules with Nrf2401 as the core in the market (Peng et al., 2017; Wang et al., 2016; Zhang et al., 2015; Ji et al., 2017; Ren et al., 2014). Differentiating from the communication distance, the modules can be divided into categories of from 10 meters to 200 meters, and even to a greater distance. And such diversity in communication distance offers broader selection for the users. Besides, some modules are supported with a solidified microcontroller, and some contain a computer port and can be used directly.

The subsequent processing of data in the microsystem can be operated on a laptop or desktop PC. After the data frames sent by the four relays are received, the indicators showing current motion status of the runner, such as the immediate heart rate, average heart rate, speed, distance, etc., are displayed on the screen by means of data and curves. By building up a databank covering information including age, gender, health status, exercise capacity, various parameters related to health and exercise effects, such as the energy consumption, longitudinal exercise ability comparison, lateral exercise ability comparison, effective heart-rate control range, etc., can be computed out. In this way, it is feasible to optimize the exercise plan in real time for the purpose of safety monitoring and scientific exercise.

\section{The Experiment Using the Heart Rate and Speed Monitoring Equipment}

To verify the basic performance of the system, the researchers conducted an application test on this system. Both "traditional manual stopwatch timing" and the "synchronous real-time monitoring device for heart rate and movement speed in longdistance running" were used.

\subsection{Participants of the Experiment}

8 long-distance running lovers from Tsinghua University voluntarily enrolled, including 6 male students and 2 female students.

\subsection{Equipment of the Experiment}

Apart from 4 sets of manual chronograph stopwatches, the researchers used one set of the synchronous real-time monitoring device for heart rate and movement speed in longdistance running. Four wireless relays were evenly distributed on the inner side of the first runway of the athletic field. The positions of the relays included the starting point of 200 meters (B Point), the midpoint of 200 meters (point $C$ ), the starting point of 300 meters (point A), and the end (point D). The distance between relays was 100 meters, as shown in Figure 2.

\subsection{Experimental Method}

4 timekeepers respectively stood at the same position in each relay, as shown in Figure 2.

\subsubsection{Manual stopwatch timing}

After commander gave the order, four timekeepers started timing with the stopwatches at the same time. When the participants reached each 100-meter timing 
A Pilot Study on Real-time Monitoring of Heart Rate and Movement Speed in Middle-distance Race of

Physical Education Classes

position, the timekeeper at that position recorded the current grades. At the end of 800 meters, the recorder A recorded the performances of the participants running 100 meters and 500 meters. The recorder B recorded the results of the participants when they completed the running of 200 meters and 600 meters. The recorder $\mathrm{C}$ recorded the results of the participants when they finished running of 300 meters and 700 meters, and the recorder $\mathrm{D}$ recorded the results that the participants performed in the running of 400 meters and 800 meters. These eight participants were tested separately and the time they spent on each 100-meter running was recorded.

\subsubsection{Automatic timing}

Based on the wireless signal transmission characteristics of Polar heart rate sensor, when the participants wearing the heart rate sensor reached near the relay, the relay received the heart rate data frame of 4 frames per second sent by the heart rate sensor and also acquired the immediate time that the participants passed by the relay. However, when the participants were more than 4 meters away from the relay, the heart sensor was out of the scope of the wireless transmission radius, and thus the relay failed to receive the data frames. Accordingly, the relay transmitted 1 to several data frames to the terminal-end computer when each participant passed by the relay.

To ensure the uniformity and accuracy of the experiment, we chose the last data that the relay received at the moment that the participants passed through each relay as the results recorded by the machine.

\subsubsection{Experiment data}

The time-recorded results by two different test methods were obtained through sorting the data, as shown in Table 3.

Table 3. Comparison of manual timing and equipment automatic timing data

\begin{tabular}{|c|c|c|c|c|c|c|c|c|}
\hline & $\begin{array}{l}\text { Male } \\
\text { No.1 }\end{array}$ & $\begin{array}{l}\text { Male } \\
\text { No. } 2 \\
\end{array}$ & $\begin{array}{l}\text { Male } \\
\text { No.3 }\end{array}$ & $\begin{array}{l}\text { Male } \\
\text { No.4 }\end{array}$ & $\begin{array}{l}\text { Male } \\
\text { No.5 } \\
\end{array}$ & $\begin{array}{l}\text { Male } \\
\text { No.6 }\end{array}$ & $\begin{array}{c}\text { Female } \\
\text { No.1 }\end{array}$ & $\begin{array}{c}\text { Female } \\
\text { No. } 2\end{array}$ \\
\hline Stopwatch test of $100 \mathrm{~m}$ & $17 " 17$ & $23 " 97$ & $27 " 98$ & $24 " 23$ & $17 " 56$ & $21 " 59$ & $25 " 17$ & $23 " 83$ \\
\hline Device test of $100 \mathrm{~m}$ & $17 " 42$ & $23 " 99$ & $28 ” 14$ & $24 " 46$ & $17 " 67$ & $21 " 70$ & $25 ” 27$ & $23 " 83$ \\
\hline Stopwatch test of $200 \mathrm{~m}$ & $35 " 90$ & $50 " 40$ & $55^{\prime \prime} 24$ & $50 " 91$ & $36 " 14$ & $46 ” 23$ & $52 " 63$ & $48 " 79$ \\
\hline Stopwatch test of $300 \mathrm{~m}$ & $53 ” 25$ & $1 ’ 13 ” 72$ & $1 ' 18 ” 64$ & $1 ’ 13 ” 34$ & $52 ” 08$ & 1’09"'82 & $1 ' 18 ” 93$ & $1 ’ 10 " 46$ \\
\hline Device test of $300 \mathrm{~m}$ & $3 ” 34$ & 1'13”80 & 1'18'79 & 1’13”39 & $51 ” 25$ & 1’09”90 & 1’19”05 & 1 1'10"61 \\
\hline Stopwatch test of $400 \mathrm{~m}$ & $1 ’ 13 ” 73$ & $1 ’ 40 ” 86$ & $1 ’ 43 ’ 35$ & $1 ' 41 ’ 97$ & $1 ' 10 ” 32$ & 1'32"69 & 1'47’65 & 1'38'72 \\
\hline Device test of $400 \mathrm{~m}$ & 1'13”91 & $1 ’ 40 ” 86$ & $1 ’ 43 ” 45$ & $1 ’ 42 ” 22$ & $1 ’ 10 ’ 35$ & 1'32"78 & $1 ’ 47^{\prime} 71$ & 1'38'74 \\
\hline Stopwatch test of $600 \mathrm{~m}$ & 2’01”99 & 2’37’'26 & 2’33”63 & 2’41”48 & 1’49”31 & 2’26”64 & 2'58'28 & 2’39’79 \\
\hline Device test of $600 \mathrm{~m}$ & 2’02”01 & 2’37’34 & 2’33”81 & 2’41”56 & 1'49”36 & $2 ’ 26 ’ 72$ & 2'58’35 & 2'40”04 \\
\hline Stopwatch test of $700 \mathrm{~m}$ & $2 ’ 23 ” 29$ & 3'00"'65 & 2’54”'17 & 3’04’76 & 2’06”74 & $2 ’ 47 ” 68$ & 3'24'’72 & 3'04'"18 \\
\hline Device test of $700 \mathrm{~m}$ & $2 ’ 23 ” 50$ & 3'00"68 & 2'54”26 & 3'04'78 & 2’06”92 & $2 ’ 47 ’ 79$ & 3’24’'87 & 3’03'”23 \\
\hline Stopwatch test of $800 \mathrm{~m}$ & $2 ’ 45^{\prime \prime} 62$ & 3'25'19 & 3'17'46 & 3'22'"84 & 2’24”84 & 3'14'87 & 3'52”'87 & 3'32"63 \\
\hline Device test of $800 \mathrm{~m}$ & $2 ’ 45 ’ 72$ & 3’25’39 & 3'17'"59 & 3'23”05 & $2 ’ 25 ’ 07$ & 3'15''12 & 3'52’'89 & 3'32'78 \\
\hline
\end{tabular}




\subsection{Results of Experiments and its Analysis}

It is shown in Table 3 that both the device timing and manual stopwatch timing presented a certain delay. We ake the male participants No. 2 and female participant No. 1 as the examples. In view of the results by stopwatch timing as the standard, the results obtained by the device timing was subtracted from the results of the manual stopwatch timing, and then the difference of the male No. 2 between the two timing methods and the difference of the female No. 1 between the two timing methods were obtained, as shown in the Figure 3.
As can be seen from the analysis in Figure 3, for male No. 2 and female No. 1, the differences between the manual stopwatch timing and device timing for 100 meter running fell into the range of $0 \sim 0.25$ seconds. According to the feature that the heart rate sensor transmitted 4 data frames per second, the relay received 1 frame of data every 0.25 seconds. The difference within 0.25 seconds was in conformity with the feature of data sending. Thus, the precision of the device timing meets the need of long-distance running monitoring.

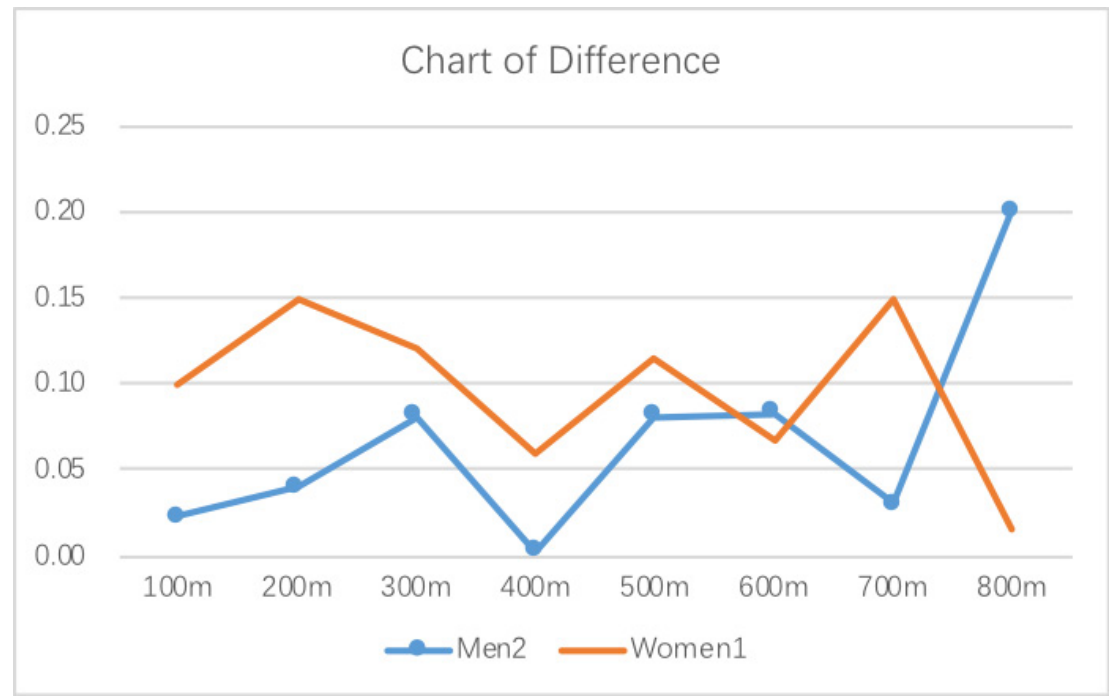

Figure 3. Chart of difference in results between manual timing and device timing

\section{Conclusion}

Practices have proved that the middledistance running carried out in universities is an activity that can effectively enhance the mental and physical health of students. During the exercise process, it is of great importance to focus on the scientific nature of the exercise, so that each individual can embrace an efficient exercise result. An insufficient amount of exercise cannot reach up to a reasonable and effective exercise stimulation intensity, while an excessive amount of exercise might damage the body. In response to the fact, we pursue a scientific, reasonable and effective way of exercise. Therefore, monitoring the exercise load of runners in real time through heart rate, as well as the 
A Pilot Study on Real-time Monitoring of Heart Rate and Movement Speed in Middle-distance Race of Physical Education Classes

movement track through speed and distance is of crucial significance. In this way, we can provide a full-aspect online scientific guidance and safety supervision on the whole motion process.

Starting from the perspective of cluster monitoring and guidance, this study focuses on the application level of micro system designing. The features of Polar heart rate sensor transmitting for wireless signals have also been emphasized. After conducting an in-depth investigation on the wireless relays of heart rate sensor, as well as the realtime follow-up processing, the researchers of this study have achieved a synchronous monitoring on the heart rate and speed in the process of long- and middle-distance running. Furthermore, the real-time remote monitoring has also been achieved through the application of wireless relay.

The application experiment shows us that this set of micro system characterized with a simple approach and sensible designing is able to satisfy the requirement of long-distance running exercise. It imposes a strong practical value to the safe monitoring, the reasonable exercise deployment, the controlling of sporting intensity, as well as the scientific exercise in the process of long-distance and middle-distance running. Therefore, it can provide an objective and effective reference basis for the middle-distance physical education class in universities.

\section{Acknowledgements}

This study is supported by the National Social Science Fund. It is part of a larger research project entitled "Research on the Monitoring Methods of Long-Distance Race Load Based on Network Cluster Technology". This project is a general project of the National Social Science

\section{References}

Augustyniak, P. (2011). Wearable wireless heart rate monitor for continuous longterm variability studies. Journal of Electrocardiology, 44(2), 195-200.

Han, G., Gu, W., \& Zhu, J. (2017) Development of a portable ECG monitor based on BMD101 chip. Chinese Journal of Medical Physics, 34(8), 850-854.

Ji, X., Zhao, C., Ning, C., Zhang, T., Gu, J., \& Tian, F. (2015). Design of Heart Rate Variability Analysis System Based on BMD101. Journal of Changchun University of Science and Technology (Natural Science Edition), 38(5), 158-162.

Ji, Y., Shi, Z., Ren, J., \& Wang, C. (2017). Three-dimensional velocity measurement of high-speed rotating sphere based on monocular vision servo system. China Sport Science and Technology, 53(2), 139144.

Jiang, Y., Huang, H., He, W., \& Chen, Y. (2015). A design of wearable ECG monitoring system based on zigBee. Microcomputer \& Its Applications, 34(16), 83-86.

Lows, SA. \& Ólaighin, G. (2014). Monitoring human health behavior in one's living environment: a technological review. Medical Engineering and Physics, 36(2), 147-168.

Neurosky. (2018, Oct 1). To provide ECG and EEG solutions for wearable devices. Retrieved from http://www.neurosky.com. $\mathrm{cn} /$

Peng, L., Lin, J., \& Pang, Y. (2017). Wearable ECG signal detection system based on adaptive filtering. Application of Electronic Technique, 43(9), 17-21.

Ren Y., Fang Z., Xu, D., \& Tan, M. (2014). Classification of table tennis rotary flight path patterns based on fuzzy neural network. Control and Decision, 29(2), 
263-269.

Wang, P., Zhang X., \& Fu, L. (2016). Design and integration of real-time monitoring device for exercise heart rate and motion action. Chinese Journal of Rehabilitation Theory and Practice, 22(1), 23-26.

Yang, K., Cong, L., Hu, W., Xu, W., Hui, D., \& Song, B. (2014). Embedded wireless ECG monitoring system based on BMD101. Electronic Technology Application, 40(1), 122-124.

Yokota, T. (2015). An experimentation of wearable low-cost sensor detecting relative amount of perspiration. Biophysical Journal, 93(6), 1923-1937.

Zhang, W., Wang, P., Fu, L., \& Cai, J. (2015). Development of exercise heart rate acquisition technology. Sensors and Microsystems, 34(12), 1-4 Turn 8.

Zhang, X., Wang, P., \& Fu, L. (2015). Design of wireless relay for rate sensor in physical exercise center. Sensors and Microsystems, 34(12), 101-103 Turn 107.

\section{Contact the Author}

\section{Daqing $Z h u$}

Tsinghua University

Hubei University of Arts and Sciences

Email: xfzdq@163.com

\section{Xingui Zhang}

Tsinghua University

Email: zxg@tsinghua.edu.cn

\section{Lanying $\mathrm{Fu}$}

Zhengzhou Shengda Economic and Trade Management College

Email: lanying@126.com

\section{Peiyong Wang}

Professor

Tsinghua University

Email: wangpy@tsinghua.edu.cn

\section{Shaohong Zhai}

Xi'an Physical Education University

Email: 416900526@qq.com 